\title{
Expansion velocities of selected PNe
}

\author{
Silvia Torres-Peimbert ${ }^{1}$, Anabel Arrieta ${ }^{2}$, and Leonid Georgiev ${ }^{1}$ \\ ${ }^{1}$ Instituto de Astronomía, Universidad Nacional Autónoma de México, Mexico \\ email: silvia@astro.unam.mx \\ ${ }^{2}$ Universidad Iberoamericana, Mexico, email: arrieta@uia.edu
}

\begin{abstract}
In order to clarify the observed relation between the expansion velocity measured from lines of different ions and their ionization potential, we present kinematical data for several objects. We have measured radial velocities on ESO UVES high dispersion spectra to compare expansion velocities for a set of planetary nebulae.
\end{abstract}

Keywords. planetary nebulae: individual (Me1-1, NGC 6153, Me1-42, Hf2-2), techniques: spectroscopic

\section{The problem}

There is a standing controversy on the explanation of the different chemical abundances of the heavy elements derived in planetary nebulae from optical recombination lines and those derived from forbidden lines, or collisionally excited lines. There are two schools of thought to solve this controversy.

I. That the nebular material is a chemically homogeneous medium with strong temperature and density variations, where the optical recombination lines depend to a first approximation like $1 / \mathrm{T}$ favoring the low temperature regions and consequently are represented by a temperature lower than the average while the collisionally excited lines that depend on $\exp (-E / k T)$ favoring the high temperature regions and are represented by a temperature higher than the average one (e. g. Peimbert 1967; Peimbert \& Peimbert 2006, and references therein).

II. Alternatively, that many $\mathrm{PNe}$ are chemically inhomogeneous and can be represented by the so called two-abundance nebular model (e.g. Liu 2006, and references therein), that can be characterized by two components: (a) a low density component, where most of the mass is relatively hot and emits practically all the intensity of the $\mathrm{H}$ lines and of the visual and UV forbidden lines, and part of the intensity of the He I lines, and (b) a high density component, with only a small fraction of the total mass, which is relatively cool, H-poor, and rich in heavy elements, and emits part of the He I and of the recombination line intensities of the heavy elements but practically no $\mathrm{H}$ and no heavy element collisionally excited lines.

\section{Results}

In order to advance on this problem, we have studied the kinematical behavior of the emission lines of a set of planetary nebulae observed with ESO UVES at high resolution, that are available for public use. In the first case we expect the expansion velocity of the O II lines to be similar to that of the of other emission lines of different ionization potential. In the second case the expansion velocities of the permitted and forbidden lines might show different behavior.

We have selected those objects that have two component lines (corresponding to the separate componentes of the receding and approaching gas) with significant $\mathrm{S} / \mathrm{N}$ (namely, 

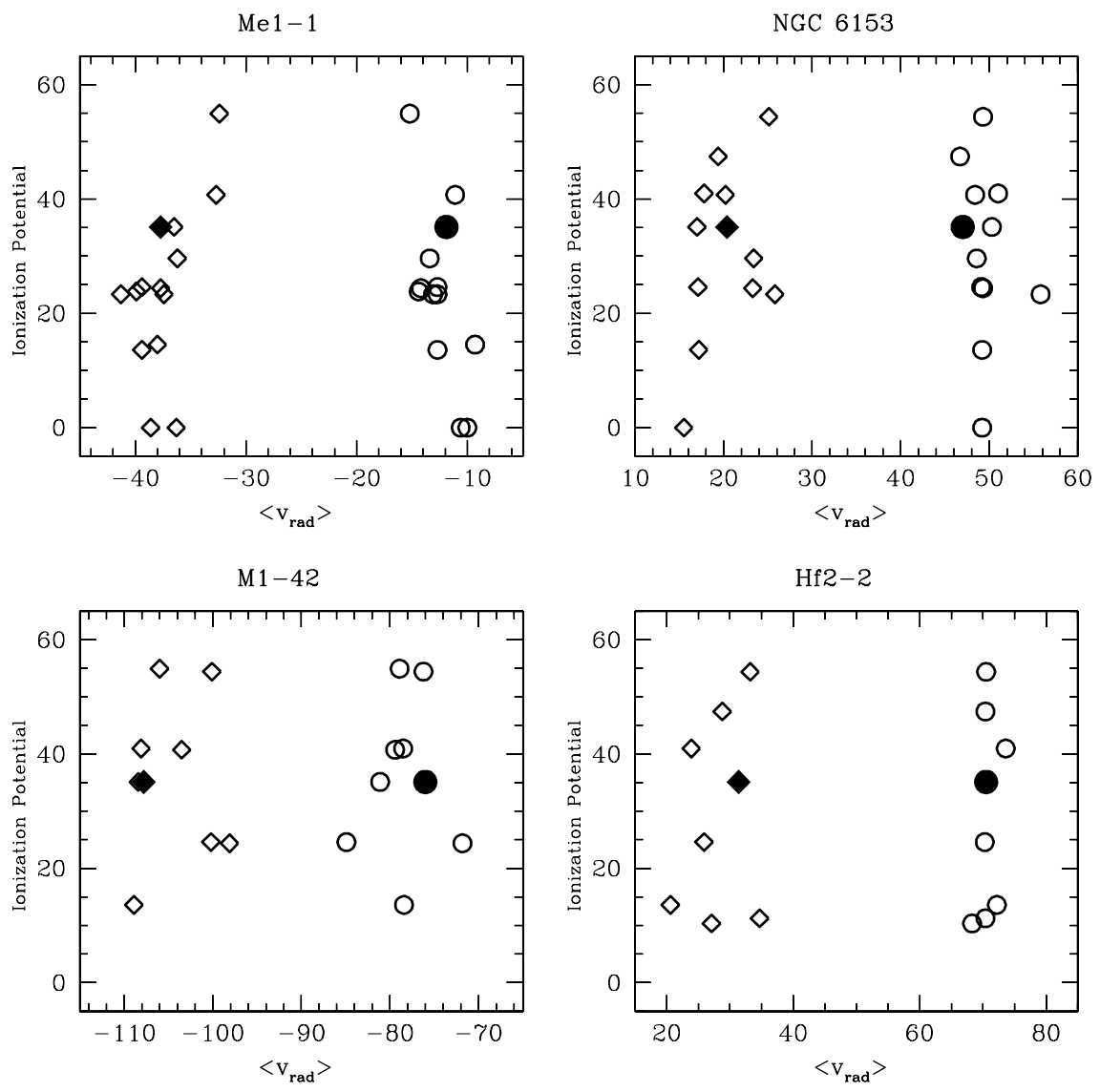

Figure 1. Blue and red components of emission lines. Solid symbols correspond to O II lines.

Me1-1, NGC 6153, M1-42, and Hf2-2). The comparison is presented in Fig. 1. We find no significant difference between the kinematical behavior of the $\mathrm{O}$ II lines relative to the rest of the emission lines (both permitted and forbidden ones). In the case of Me1-1 we find a significant trend of lower expansion velocity rate for the high ionization species relative to lower ionization ones, which is to be expected due to the ionization stratification within the nebula (e. g. Bohuski \& Smith 1974, and references therein).

We plan to continue this project including all the available material, and to extend it to compare in detail the position-velocity diagrams of the $\mathrm{O}$ II and [O III] lines in the original echellograms.

\section{Acknowledgements}

We are grateful to CONACyT 129753, 106719 and DGAPA-UNAM IN111708 projects. This work was based on data obtained from the ESO Science Archive Facility.

\section{References}

Bohuski, T. J. \& Smith, M. G. 1974, ApJ, 193, 197

Liu, X.-W. 2006, IAU Symposium 234, Planetary Nebulae in our Galaxy and Beyond, M. J. Barlow \& R. H Mendez (eds.), Cambridge, p. 219

Peimbert, M. 1967, ApJ, 150, 825

Peimbert, M. \& Peimbert, A. 2006, IAU Symposium 234, Planetary Nebulae in our Galaxy and Beyond, M. J. Barlow \& R.H Mendez (eds.), Cambridge, p. 219 